\title{
Research on Rotational Resistance Moment of Rotary Energy Recovery Device
}

\author{
Ang GUO ${ }^{1}$, Zuo-dong $\mathrm{HUANG}^{1}$, Zai-ran $\mathrm{DING}^{l}$, Yi $\mathrm{SHI}^{1}$, Lei $\mathrm{JIAO}^{1 *}$ and Jian-xing $\mathrm{LENG}^{l}$ \\ ${ }^{1}$ Institute of Naval Architecture and Ocean Engineering, Zhejiang University, Zhoushan, China, 316000
}

\begin{abstract}
The effects of operational conditions on the resistance moment of a rotary energy recovery device have been systematically investigated through the computational fluid dynamics in this paper. Based on the structural characteristics of the rotary energy recovery device, the hydro-static bearing was established on both sides of the rotor. Simulation results indicate that for the rotation resistance moment generated by the rotor internal flow path, the rotation speed is linearly positive with the rotational resistance moment. For the rotation resistance moment generated by the clearance flow, the rotation speed is also positively correlated with the rotational resistance moment. The circular clearance size is negatively correlated with the rotation resistance moment, and the endcover fluid film size is negatively correlated with the rotation resistance moment. The boundary speed between laminar flow and turbulent flow was found by comparing the changing trend of rotational resistance moment. The obtained relation between clearance, the speed of the rotor and resistance moment provides a simple way to predict the resistance moment of the device, which will be beneficial to design the energy-efficient rotary energy recovery device
\end{abstract}

\section{Introduction}

Seawater reverse osmosis (SWRO) technology is now a well established water desalination way to solve the water shortage problem globally[1-4]. The rotary energy recovery device (RERD) can effectively recover the pressure energy from the rejected high pressure brine on the principle of positive displacement and has become one of the key facilities to reduce the operational cost in SWRO system[5,6]. When the rotor of RERD is rotated, the performance of the energy recovery device impact is greatly affected by the endcover clearance, circular clearance,and rotate speed of the rotor. As is known, a small clearance could bring a good seal performance by making the leakage passage much more narrow. However, when the mating clearance is decreased to an extremely small level, the friction pair may work in the state of contact friction[7], which will raise the energy consumption of the energy recovery device and thus lessen efficiency of the device. Therefore, finding a proper way to keep the rotor working in the fluid film lubrication condition at a small clearance becomes the key aim in the RERD field[8].

In this paper, to find the appropriate clearance and research the effect of speed, circular clearance and endcover clearance how to affect the performance of energy recovery device, a series of clearance flow models were established, through changing the endcover clearance,circular clearance and adjusting the speed of the rotor, and then CFD is used to analyze the resultant rotation resistance moment at different fluid film thickness and rotate speed.

\section{Working principle of the RERD}

As seen in Fig.1, the core components of the RERD consist of the sea-water endcover, the rotor, the rotor sleeve and the brine endcover. The seawater endcover contains a port for incoming low pressure seawater (LP-IN), and a port for outgoing high pressure seawater (HP-OUT). The brine endcover likewise embodies two ports (HP-IN and LP-OUT)[9]. The rotor containing axial ducts arranged in a circle is fit into a rotor sleeve between the seawater endcover and the brine endcover. The rotor is the only rotating part of the device[10].

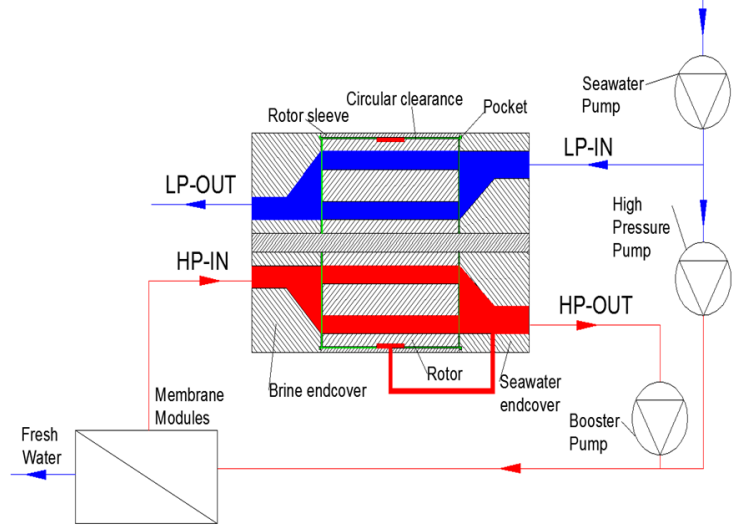

Fig. 1. Schematic representation of the SWRO and RERD

\footnotetext{
*Corresponding author: jiaolei@zju.edu.com
} 


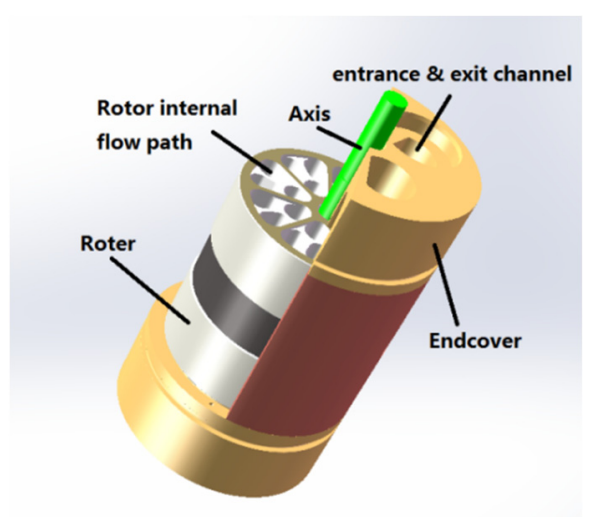

Fig. 2. geometric model of RERD

The geometric model of rotary energy recovery device is shown in Fig.2. Every endcover has two channels, one is entrance, the other is exit. In addition, there have many flow paths in the rotate.

In order to research the effect of speed, circular clearance and endcover clearance on the moment of rotation resistance, we draw a series of clearance flow models to analyze. The smallest clearance is $0.01 \mathrm{~mm}$, the maximum clearance is $0.05 \mathrm{~mm}$ and several clearance flow models are established at each interval of $0.01 \mathrm{~mm}$. That is, 5 parameters of endcover clearance and 5 parameters of circular clearance, a total of 25 models. The main characteristic parameters of the geometric model are shown in the Table.1.

Table.1. The main parameters of the geometric model

\begin{tabular}{cc}
\hline parameters & numerical value \\
\hline outer diameter of therotor & $190 \mathrm{~mm}$ \\
inner diameter of the rotor & $28 \mathrm{~mm}$ \\
height of the rotor & $190 \mathrm{~mm}$ \\
height of the endcover & $100 \mathrm{~mm}$ \\
endcover angle & $22^{\circ}$ \\
endcover clearance & $0.01 、 0.02 、 0.03 、 0.04 、$ \\
& $0.05 \mathrm{~mm}$ \\
circular clearance & $0.01 、 0.02 、 0.03 、 0.04 、$ \\
& $0.05 \mathrm{~mm}$ \\
\hline
\end{tabular}

Rotate speed is set respectively as $300 、 600 、 750$ 、 900、1200、1500、1800 rpm, total 7 computing points.

Based on the data collected from the experiment, the boundary conditions at the inlet of high and low pressure are set as Table.2.

Table.2. Import and export boundary condition setting

\begin{tabular}{|c|c|c|}
\hline & boundary conditions & numerical value \\
\hline HP-IN & velocity-inlet & $8.1 \mathrm{~m} / \mathrm{s}$ \\
\hline HP-OUT & pressure outlet & $6 \mathrm{Mpa}$ \\
\hline LOW-IN & velocity-inlet & $8.1 \mathrm{~m} / \mathrm{s}$ \\
\hline LOW-OUT & pressure outlet & $0.3 \mathrm{Mpa}$ \\
\hline
\end{tabular}

\section{Analysis of simulation results}

In this paper, the simulation is divided into two parts, one is rotation resistance moment generated by internal flow of rotor, just considering the rotation speed. The other part is the rotational ${ }^{i}$ resistance moment generated by the clearance flow, considering the rotation speed, circular clearance and endcover clearance between the three variables, so by changing one of the variables to analyze the impact of changes in the rotation torque variable.

\subsection{The rotational resistance moment in the inner runner of the rotor varies with the rotational speed.}

The process of energy recovery occurs inside the rotor, with a large amount of fluid inside the rotor. So, the rotation resistance moment generated by the inner runner of the rotor cannot be ignored.

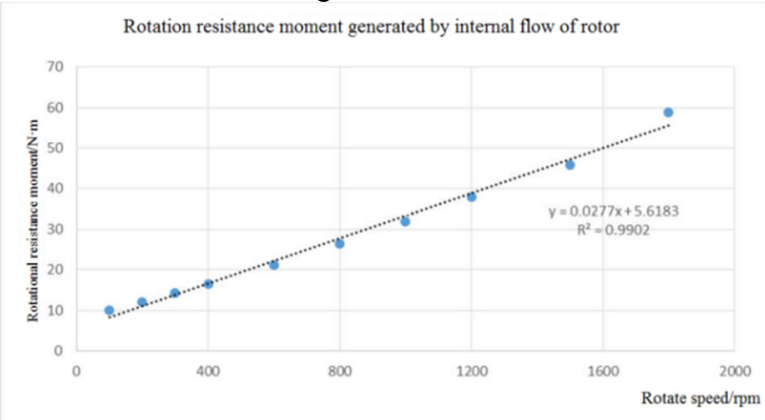

Fig.3. Rotation resistance moment (Inside the rotor) with rotational speed

In the Fig.3, the rotation resistance moment of rotor flow generated by numerical simulation is obtained with the XY scatter diagram of different rotational speed. The greater the rotor speed is, the greater the rotation resistance moment is. Obviously, the rotational resistance moment has a significant positive linear correlation with the rotor speed. At the same time, due to the obvious regularity of scatter data distribution, the regression model can be applied to analyze. We use a linear function to fit the scatter points, the regression function formula is obtained by regression analysis:

$$
y=0.0277 x+5.6183
$$

In this equation, $y$ represents the rotational resistance moment generated by the rotor runner. $x$ represents the rotor speed, and fitting coefficient R2 is 0.9902 .

\subsection{The effect of the endcover clearance on the rotational resistance moment in the clearance flow.}

According to Newton's theory of internal friction in fluid dynamics, for the same liquid, the shear stress is proportional to the velocity $\mathrm{U}$, and is inversely proportional to the spacing $\mathrm{h}$ of the two plates. Therefore, the change of the endcover clearance is equal to the change of the plate spacing $h$, which directly changes the shear stress of the rotating rotor end to the surface, thus affecting the size of the torque of the rotational resistance moment. 
In the Fig.4, the rotational resistance moment generated by the clearance flow and the numerical simulation are shown in the XY scatter diagram with different endcover clearance and rotate speed. Obviously, the rotational resistance moment has a positive correlation with the rotor speed, which is approximately linear. The greater the rotational speed is, the greater the rotation resistance moment generated by the clearance flow is.

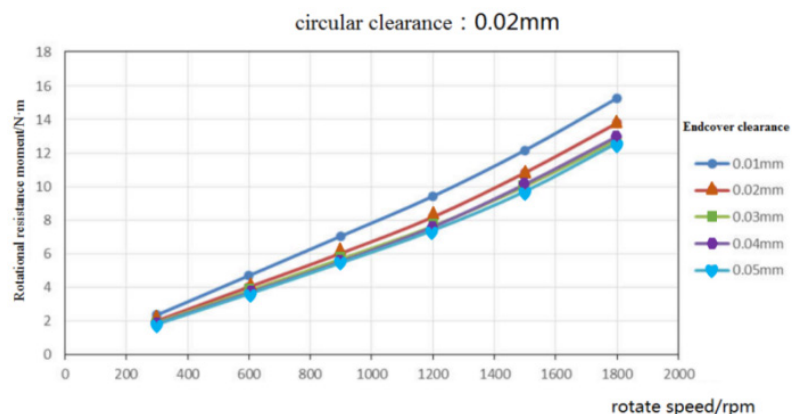

Fig.4. Relation between Rotational resistance moment and rotate speed

For a fixed type of rotor, the change of rotational speed determines the relative linear velocity of the clearance flow wall at both sides. According to Newton's theory of internal friction in fluid dynamics, for the same liquid, wall shear stress $\tau$ is proportional to the relative velocity of the wall. So, in the range of 300 1200 rpm, the rotation resistance moment curve of each endcover clearance is linear relationship. When the speed is greater than $1200 \mathrm{rpm}$, the change curve shows an obvious uptrend. It can be concluded that when the speed is less than 1200rpm, the fluid in the clearance flow is laminar flow, and when the speed is greater than $1200 \mathrm{rpm}$, the fluid in the gap flow is gradually changed into turbulent motion.

At the same time, at low speed, such as $300 \mathrm{rpm}$, the rotation resistance moments under different ends are very close. However, with the gradual increase of the rotational speed, the rotation resistance moments of the different ends are differentiated. The rotation resistance moment of the endcover clearance is $0.01 \mathrm{~mm}$ and 0.02 $\mathrm{mm}$, which is significantly larger, while the other growth rate is almost the same. Fig.5 is to better analyze the effect of the change of endcover clearance on the rotational resistance of clearance flow.

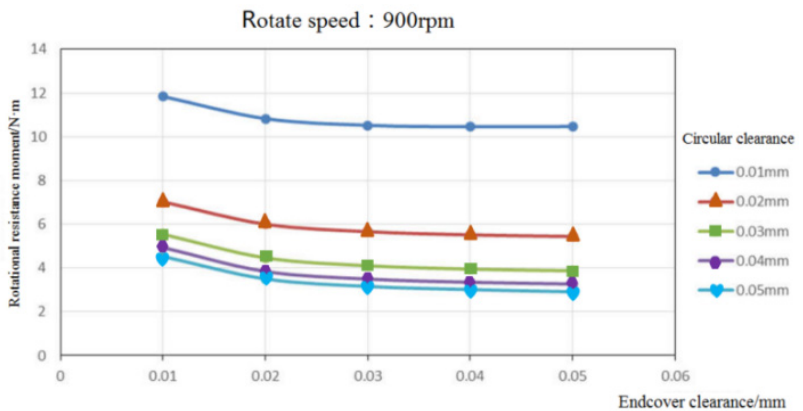

Fig.5. Relation between Rotational resistance moment and endcover clearance

In the Fig.5, the rotational resistance moments generated by the clearance flow and the numerical simulation are shown in the $\mathrm{XY}$ scatter diagram with different endcover clearance and different circular clearance. In order to highlight the change of the curve, the speed of 900 RPM is selected. It can be seen that under the rotation speed, the torque of the rotational resistance decreases with the increasing of the endcover clearance. After careful observation, it can be found that the change curve of the rotation resistance moment in the range of $0.01 \sim 0.02 \mathrm{~mm}$ in the endcover clearance is particularly obvious. However, the change curve of the rotation resistance moment in the range of $0.02 \sim 0.05 \mathrm{~mm}$ in the circular clearance tends to be gentle and almost flat.

According to the theory of Newton's9 internal friction in fluid dynamics, the shear stress of the wall $\tau$ is inversely proportional to the distance $\mathrm{h}$ between the two walls. Therefore, when the endcover clearance is less than $0.02 \mathrm{~mm}$, the fluid in the clearance flow is laminar motion. When the endcover clearance is greater than 0.02 $\mathrm{mm}$, the fluid in the clearance flow is gradually transformed into a turbulent motion.

\subsection{The effect of the circular clearance on the rotational resistance moment in the clearance flow.}

According to Newton's theory of internal friction in fluid dynamics, for the same liquid, the shear stress is proportional to the velocity $\mathrm{U}$, and is inversely proportional to the spacing $h$ of the two plates. Therefore, the change of the circular clearance is equal to the change of the plate spacing $\mathrm{h}$, which directly changes the shear stress of the rotating rotor ring to the surface, thus affecting the size of the torque of the rotational resistance moment.

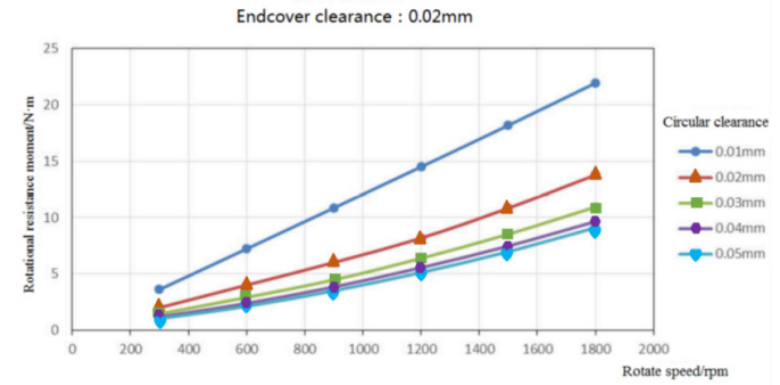

Fig.6. Relation between Rotational resistance moment and rotate speed

In the Fig.6, the rotational resistance moment generated by the clearance flow and the numerical simulation is shown in the XY scatter diagram with different circular clearance and different rotate speed. In order to highlight the change of the curve, the speed of 900 RPM is selected. In order to meet the working conditions of the rotary energy recovery device in the experiment, the endcover clearance is selected as 0.02 $\mathrm{mm}$.

Obviously, the rotational resistance moment has a positive correlation with the rotational speed, and can even be considered as a linear positive correlation. That is, the greater the rotational speed, the greater the rotation resistance moment generated by the clearance flow. For a fixed type of rotor, the change of rotational speed determines the relative linear velocity of the side walls of 
the clearance flow.

According to the theory of Newtonian internal friction in fluid dynamics, For the same liquid, the shear stress of the wall $\tau$ is proportional to the velocity $\mathrm{U}$. In the Fig.6, It is only a linear relationship with a continuous linear relationship with the rotation resistance moment of the circular clearance of $0.01 \mathrm{~mm}$. When the rotational speed is within the range of 300 1200 RPM, the rotational resistance moment change curve of each circular clearance is typical of linear relationship. When the RPM is over 1200 RPM, the change curve shows an obvious uptrend. It can be concluded that when the rotation speed is less than 1200 RPM, the fluid in the clearance flow is the laminar movement. When the RPM is greater than $1200 \mathrm{RPM}$, the fluid in the gap flow is gradually changed into turbulent motion.

At the same time, in the low speed state, such as 300 RPM, the rotation resistance moment of different circular clearances is very close to the torque. However, with the gradual increase of the rotational speed, the rotation resistance moments of different circular clearances are differentiated. The rotation resistance moment at $0.01 \mathrm{~mm}$, $0.02 \mathrm{~mm}$ and $0.03 \mathrm{~mm}$ was significantly increased, while the growth rate of the other two was almost the same. In order to better analyze the effect of the change of the circular clearance on the rotation resistance moments of the clearance flow, we continue to observe Fig.7. Rotate speed:900rpm

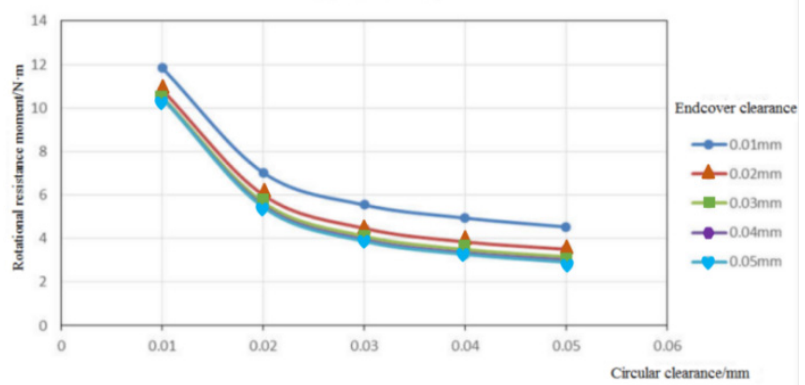

Fig.7. Relation between Rotational resistance moment and circular clearance

In the Fig.7, the rotational resistance moment generated by the clearance flow generated by the numerical simulation is shown in the XY scatter diagram with different endcover clearance and different circular clearance. In order to highlight the change of the curve, the speed of 900 RPM was selected.

It can be seen that under the condition of different endcover clearance, the rotational resistance moment decreases with the increase of the circular clearance and presents a negative correlation. After careful observation, it can be found that the change curve of the rotation resistance moment in the circular clearance of 0.01 0.03 $\mathrm{mm}$ is especially significant. The change curve of the rotation resistance moment in the circular clearance of $0.03 \sim 0.05 \mathrm{~mm}$ was reduced flattens out.

According to the analysis of Fig. 6 and Fig. 7, it can be determined that, when the circular clearance is less than $0.03 \mathrm{~mm}$, the circular clearance has obvious influence on the rotational resistance moment generated by the clearance flow, the influence is negative correlation. The larger the circular clearance, the smaller the rotational resistance moment. In the case that the circular clearance is greater than $0.03 \mathrm{~mm}$, the effect of the circular clearance on the rotational resistance moment generated by the clearance flow is rapidly weakened. According to the theory of Newtonian internal friction in fluid dynamics, the shear stress of the wall $\tau$ is inversely proportional to the distance $\mathrm{h}$ between the two walls. So, When the circular clearance is less than $0.03 \mathrm{~mm}$, the fluid in the clearance flow is laminar motion. When the circular clearance is greater than $0.03 \mathrm{~mm}$, the fluid in the clearance flow is gradually transformed into a turbulent motion.

\section{Conclusion}

(1) For the rotation resistance moment generated by the rotor internal flow path, the rotation speed is linearly positive with the rotational resistance moment, and the linear function can be applied to obtain the regression function by fitting the scattered points.

(2) For the rotation resistance moment generated by the clearance flow, the rotation speed is also positively correlated with the rotational resistance moment. The circular clearance size is negatively correlated with the rotation resistance moment, and the endcover fluid film size is negatively correlated with the rotation resistance moment.

(3) However, the effect of clearance size on the torque of rotation resistance is limited and uneven. When the circular clearance is less than $0.03 \mathrm{~mm}$, the rotation resistance moment changes rapidly. When the circular clearance is greater than $0.03 \mathrm{~mm}$, the rotation resistance moment change tends to be gentle. When the endcover clearance is less than $0.02 \mathrm{~mm}$, the rotation resistance moment changes rapidly. When the endcover clearance is greater than $0.02 \mathrm{~mm}$, the rotation resistance moment change tends to be gentle, even without impact. In comparison, the effect of the circular clearance on the moment of rotation resistance is more obvious than that of the endcover.

\section{References}

1. A. Ghobeity, A. Mitsos, Desalination 263 (2010) 7688.

2. B. Qi, Y. Wang, S. Xu, Z. Wang, S.Wang, Ind. Eng. Chem. Res. 51 (2012) 14135-14144.

3. A.Zhu, P.D.Christofides, Y. Cohen, Ind. Eng. Chem. Res. 48 (2008) 6010-6021.

4. A. Zhu, P.D. Christofides, Y. Cohen, Ind. Eng. Chem. Res. 48 (2009) 9581-9589.

5. D. Song, Y. Wang, S. Xu, Z. Wang, H. Liu, S. Wang, Desalination 348 (2014) 1-7.

6. S. Mambretti, E. Orsi, S. Gagliardi, R. Stover, Desalination 238 (2009) 233-245.

7. C.L. Brockett, P. Harper, S. Williams, G.H. Isaac, R.S Dwyer-Joyce, Z. Jin, J. Fisher, J. Mater. Sci. Mater. Med. 19 (2008) 1575-1579.

8. E. Xu, Y Wang, J. Wu, S Xu, Desalination 383 (2016) $60-67$ 
9. I.B. Cameron, R.B. Clemente, Desalination 221 (2008) 136-142.

10. R.L. Stover, Desalination 221 (2008) 126-135. 